THE KNOWLEDGE RESOURCE BASE SERIES /

LA SÉRIE « BASE DES RESSOURCES DES CONNAISSANCES »

\title{
THE KNOWLEDGE RESOURCE BASE SERIES
}

\author{
Editor's Introduction
}

The following paper is one in a series focusing on new ways of thinking about how we understand and make sense of mental illness. Katherine Boydell and her colleagues explore the similarities and differences between professional perspectives and those of consumers/survivors and families, and report on focus groups designed to confront the issues face to face. This work is centered on the Knowledge Resource Base (KRB), a concept that maps four various categories of knowledge: medical/clinical, social science, experiential, and cultural/traditional. The KRB attempts to move away from seeing any one of these categories as necessarily privileged in its ability to explain the phenomenon of mental illness as experienced in various human societies; instead, it calls for an ongoing dialogue between and among them. A dialogue informed by this rich base of understanding will, it is believed, produce the best possible working understanding of mental illness.

The Editors of the Canadian Journal of Community Mental Health invite further papers on the Knowledge Resource Base. Interested authors can see the original call for papers and concept paper in the Spring 1997 issue. Papers that explore any one aspect of the concept or interactions between them, or that explore new ways to conceptualize the issue are welcome. Papers by consumers/survivors and family members are also welcome. Papers should be submitted to:

John Trainor, Associate Editor

Canadian Journal of Community Mental Health

c/o The Centre for Addiction and Mental Health

1001 Queen St. West

Toronto ON M6J 1HA

\section{LA SÉRIE « BASE DE RESSOURCES DES CONNAISSANCES *}

\author{
Introduction du rédacteur de la série
}

L'article qui suit est le premier d'une série de textes axés sur les nouvelles façons de considérer notre compréhension et notre rationalisation de la maladie mentale. Katherine Boydell et ses collègues y analysent les similarités et les différences entre la perspective des professionnels et professionnelles et celle des personnes ayant un vécu psychiatrique et des familles. Les auteures font également rapport sur les groupes de discussion qui proposent une confrontation directe des questions soulevées. Cette recherche est fondée sur le concept de «base de res- 
sources des connaissances» (Knowledge Resource Base-KRB), qui répertorie quatre catégories de connaissances: les connaissances médicales ou cliniques, les connaissances relevant des sciences sociales, les connaissances tirées de l'expérience et les connaissances culturelles ou traditionnelles. L'approche KRB cherche à se distancer du principe qui fait de l'une ou l'autre de ces catégories l'explication la plus plausible du phénomène de la maladie mentale tel qu'il se vit dans diverses sociétés humaines; cet approche tend plutôt à privilégier l'établissement d'une interrelation, d'un échange continu entre les catégories de connaissances. Elle repose sur la ferme conviction que les échanges inspirés de cette riche base de ressources mèneront à la meilleure compréhension pratique possible de la maladie mentale.

La rédaction de la Revue canadienne de santé mentale communautaire sollicite des articles supplémentaires concernant l'approche KRB. Les auteures et auteurs intéressés peuvent prendre connaissance de l'invitation à présenter des communications et de l'article conceptuel parus dans le numéro du printemps 1997. Nous aimerions notamment recevoir des articles sur les différents aspects du concept et sur l'interaction entre ces aspects, ou des textes présentant de nouvelles manières de conceptualiser la question. Les articles rédigés par les personnes ayant un vécu psychiatrique ou les membres des familles sont également bienvenus. Veuillez faire parvenir vos soumissions à:

John Trainor, Rédacteur associé

Revue canadienne de santé mentale communautaire

À l'attention de: The Centre for Addiction and Mental Health

1001 Queen St. West

Toronto, ON M6J 1H4 